ist die Kohle schwer restlos aus dem Paraffin zu entfernen. Deshalb ist Schüttfiltration durch Kohle vorzuziehen, wobei die unterste Lage aus Kieselgur besteht. - Gerade die aktivste Kohle läßt sich am schlechtesten von den Verunreinigungen wieder befreien. Es kommt dabei aber auch sehr darauf an, ob es sich um eine physikalische oder um eine Chemosorption handelte. - Zur Regenerierung gebrauchter Schmieröle sind Bleicherden den Kohlen vorzuziehen. - Zur Entfärbung der (in Benzin usw. gelösten) Harze mischt man Bleicherde und Kohle.

$$
\text { R. E. Lg. }
$$

\section{m) Nahrungsmittelchemie}

Deuel, H., Oxydativer Abbau von Pektin in wässeriger Lösung. (Helv. chim. Acta 26, 2002, 1943.) Neben der Kettenlänge bestimmt auch der Veresterungsgrad und die Gegenwart niedermolekularer Elektrolyte den Zähigkeitsgrad hochpolymerer Pektine. $\mathrm{H}_{2} \mathrm{O}_{2}$ setzt sie irreversibel durch Oxydation herab. Lösliche Ferrosalze aktivieren diese Reaktion. Das gilt auch für Muzin. [Bei Gelatine sind Ferrisalze so wirksam. Ref.] Auch Askorbinsäure wirkt abbauend und auch seine Wirksamkeit wird durch Ferrosalz beschleunigt.

R. E. Lg.

\section{o) Filtrieren, Klären, Schlämmen, Staubbindung, Zerkleinern, Rauchfrage.}

Theilhaber, W., und H. Erlenmeyer, Uber Flotationsversuche thit 8-0xychinolin als Sammler. VI, VII. (Helv. chim. Acta 27, 1313 und 1315, 1944.)

Bei schlecht schwimmenden Eisenoxydhydratpräparaten kann die Ausbeute durch Zusatz von $\mathrm{Ni}^{++}$von 26,8 Proz. auf 92 gesteigert, durch $\mathrm{Cu}^{++}$auf 11,2 Proz. gesenkt werden. Unter etwas veränderten Bedingungen wirkt aber $\mathrm{Cu}^{++}$steigernd.

Der flotierte Anteil ist stets heller als der Rückstand. Der Unterschied wird um so größer, je wasserhaltiger der Ausgangsstoff war.

R. E. Lg.

Theilhaber, W., und H. Erlenmeyer, Uber Flotationsversuche mit Flußspat. (Helv. chim. Acta 27, $1319,1944$.

Während man mit Oxin als Sammler bei frisch gemahlenem Flußspat eine Ausbeute von 90,7 Proz. erhalten kann, fällt die Ausbeute bei einigen Wochen gelagertem auf 59 Proz. Das wird einer Oberflächenveränderung durch $\mathrm{CO}_{2}$ zugeschrieben. R. E. Lg.

Theilhaber, W., und H. Erlenmeyer, Flotationsversuche mit Pegmatit. (Helv. chim. Acta 27, $1428,1944$.

Zur Befreiung von Pegmatit von dunklen oxydischen Mineralien, wie es für die Keramik erwünscht ist, haben sich als Sammler Rindensäuren aus Föhre oder Korkeiche noch besser bewährt als das von G. Gerth 1934 empfohlene ölsaure Natrium.

R. E. Lg.

\section{q) Kunststoffe.}

Anonym, Kunstharze, ihre Herkunft und Bildung. 2. Kasein. (Plastics 6, 59, 1942.)

In Fortsetzung der Serie wird die Kaseingewinnung und Weiterverarbeitung geschildert. In einer Tabelle sind die wichtigsten Eigenschaften und gebräuchlichsten Handelsnamen zusammengestellt.

$$
\text { H.-J. Orthmann. }
$$

Staudinger, H.-Freiburg, Uber die Formbeständigkeit der Makromoleküle. (Kunststoffe 33 , 197, 1943.)

Die Arbeit ist die erweiterte Wiedergabe des vom Autor auf der Arbeitssitzung der Arbeitsgruppe:
,Physikalische und chemische Bewertung von Kunststoffen" im Dezember 1942 gehaltenen Vortrages. Sämtliche Argumente für die Formbeständigkeit organischer Makromolekeln im festen und flüssigen Zustand werden angeführt. Aus dem Viskositätsverhalten wird auf eine stäbchenförmigeForm geschlossen, jedoch ist dieses Stäbchen nicht unelastisch, sondern als „Haar" aufzufassen. H.-J. Orthmann.

\section{Bücherbesprechungen.}

Ausführung qualitativer Analysen. Von Wilhelm Biltz. Neunte Auflage besorgt von Werner Fischer. XlI/180 Seiten, mit 14 Figuren und 1 Tafel. (Leipzig 1944, Akademische Verlagsgesellschaft.) Preis geb. RM 9.60.

Die neunte Auflage dieser unübertroffenen, seit einer Generation im Unterricht bewährten Anleitung zur Qualitativen Analyse bedarf als solche fürwahr keiner empfehlenden Besprechung mehr, zumal sie gegenüber der vorangegangenen nur vereinzelte Anderungen und Ergänzungen aufweist. Wichtiger ist, hervorzuheben, daß nach dem Heimgang des Verfassers (1943) die Betreuung des Buches nunmehr sein Schüler und Amtsnachfolger Werner Fischer übernommen hat, dem die Analytische Chemie bereits wertvolle Vervollkommnungen verdankt und der bestens berufen erscheint, das meisterliche kleine Werk im Geiste seines Schöpfers fortzusetzen und auf fortschrittlicher Höhe zu erhalten. Heinrich Menzel.

Botanik II. Botanische Wanderungen. Von Dr. Johannes Richter. (Die Lehrapotheke, Band 6.) 80. VI, 75 Seiten. (Dresden 1942, Verlag Theodor Steinkopff.) Preis brosch. RM 3.50.

Während in ,,Botanik I" vom Verfasser an Hand praktischer Beispiele die Physiologie und Anatomie erklärt wird, werden im vorliegenden Buch unter Berücksichtigung der charakteristischen Merkmale der einzelnen Pflanzenfamilien die in pharmazeutischer Hinsicht wichtigsten Pflanzen besprochen. Jede indifferente Beschreibung ist dabei vermieden worden; die Tendenz des Verfassers, wenn irgend angängig, die Ableitung und Erklärung der lateinischen Namen oder Vulgärnamen zu erklären, muß besonders anerkannt werden.

H. Wojahn (Jena).

\section{Berichtigungen.}

B. RoBmann und E. Kayser:

Viskositätsmessung an wässerigen Zucker- und Natriumphosphatlösungen [Kolloid-Z. 106, 36 (1944)].

Auf Seite 37 unten, letzter Absatz, muß es richtig heißen: Neben der absoluten dynamischen Viskosität wurde auch die relative Viskosität

$$
\eta r e l=\frac{\eta c}{\eta}
$$

und die kinematische Zähigkeit

$$
v=\frac{\eta}{\varrho}
$$

sowie die spezifische Viskosität

$$
\eta \text { spez }=\eta_{\text {rel }}-1
$$

berechnet, wobei $\eta$ die Viskosität des Wassers ist 
A. Matthes:

Zur Theorie des Quellungsvorganges an Gelen [Kolloid-Z. 108, 79 (1944)].

In Kap. IV einschließlich Fig. 7 muß die B zeichnung

$$
-\frac{p-p_{0}}{A-A_{0}}
$$

durchweg ersetzt werden durch die andere Bezeichnung

$$
-\frac{p-p_{0}}{X-\bar{X}_{0}}
$$

wobei gilt

$$
X=\frac{A}{p} ; X_{0}=\frac{A_{0}}{p_{0}}
$$

Manfred v. Ardenne:

Uber ein neues Universal-Elektronenmikroskop mit Hochleistungsmagnet-Objektiv und herabgesetzter thermischer Objektbelastung [Kolloid-Z. 108, 195 (1944)].

In dieser Arbeit sind durch ein Versehen bei der Drucklegung die der Fig. 7 zugrunde liegenden Annahmen nicht mit zur Veröffentlichung gelangt. Die Annahmen lauteten:

Foliendicke $x=10^{-5} \mathrm{~mm}$

Umgebungstemperatur $t_{0}=20^{\circ} \mathrm{C}$

Strahlungszahl $C=1,2 \cdot 10^{-12} \frac{\mathrm{cal}}{\mathrm{cm}^{2} s\left({ }^{0} K\right)^{4}}$

Wärmeleitzahi $\lambda=5 \cdot 10^{-4} \frac{\mathrm{cal}}{\mathrm{cm} \mathrm{s}^{0} \mathrm{~K}}$.

P. H. Hermans, J. J. Hermans und D. Vermaas:

Uber Dichte und Lichtbrechungsvermögen von Zellulosefasern [Kolloid-Z. 109, 9 (1944)].

In der Tabelle II muß der Teil für native Fasern folgendermaßen heißen:

Tabell e' II.

\begin{tabular}{|c|c|c|c|c|c|}
\hline Faserart & $n_{\|}$ & $n_{1}$ & niso & $u$ & $\frac{n_{\text {iso }}-1}{d}$ \\
\hline 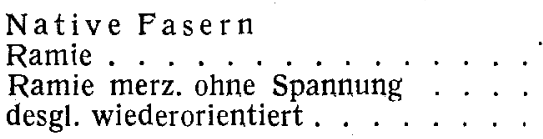 & $\begin{array}{l}1,601^{5} \\
1,587^{3} \\
1,579^{3}\end{array}$ & $\begin{array}{l}1,530^{7} \\
1,532^{5} \\
1,528^{8}\end{array}$ & $\begin{array}{l}1,554^{3} \\
1,550^{8} \\
1,545^{6}\end{array}$ & $\begin{array}{l}1,553 \\
1,546 \\
1,526\end{array}$ & $\begin{array}{l}0,3568 \\
0,3563 \\
0,3573\end{array}$ \\
\hline
\end{tabular}

Beobachtete Brechungsindizes $n_{4}$ und $n_{\perp}$ verschiedener Zellulosefasern, das Brechungsvermögen $n_{\text {iso }}$, die beobachteten Dichten bei $20^{\circ}$ und die daraus berechneten Refraktionen $\left(n_{\text {iso }}-1\right) / d$.

ferner ist bei den Sedura-Fasern unter b), c) und d) das Wort „Prozent“ einzufügen.

\section{Autoren-Register zur Kolloid-Zeitschrift, Bd. 108 und 109*) (1944)}

O. = Originalabhandlung, R. = Referat, B. = Bücherbesprechung, P. B. = Patentbesprechung. Die erste fette Zahl zeigt den Band (108 oder 109) an, die zweite die Seitenzahl.

Alexander, P., J.A. Kitchener und H.V.A. Briscoe, Wirkung von Wachsen und anorganischen Pulvern auf den Wasserdurchtritt durch Zelluloidmembranen. R. 109, 43

Alfeis, M., s. W. Scheele

Althoff, H., Kochsalzquaddelresorptionszeit. R 109,47

Altmann, R.F.A., Analyse der Hevealatex-Proteine: Identifizierung und Eigenschaften der isolierten Erzeugnisse. R, 109, 48

Ardenne, M.v., Neues Universal-Elektronenmikroskop mit Hochleistungsmagnet-Objektiv und herabgesetzter thermischer Objektbelastung. $O$. 108,$195 ; 109,162$

- Auflösungsvermögen photographischer Schichten für Elektronenstrahlung. R. 109, 115

- Eignung bindemittelarmer Photoschichten in der Elektronen-Ultramikroskopie. R. 109, 115

Bartels, J., Geophysik. B. 108, 64

Bavendamm, W., Fortschritte der Holzkonservierung. R. 108, 271
Bayerle, H., und G. Borger, Verdaulichkeit von Tumorgewebe durch normale Enzyme. R. 109, 43

Bendel; H.J., s. J. B. A. Stroink

Benedicks, C., und P. Sederholm, Einfluß einer adsorbierten Schicht auf die Kohäsion und Koaleszenz bei flüssigen und festen Körpern. R. 109,112

Bergwall, J., s. T. N. Nilsson

Beythien, A., Laboratoriumsbuch für den Lebensmittelchemiker. B. 108, 64

Biltz, W., und W. Fischer, Ausführung qualitativer Analysen. B. 109, 161

Blank, F., und H. E. Deuel, Einfluß von Heteroauxin auf die Quellung von Membransubstanzen. R. 108,60

Blaschke, F., Der übermolekulare Bau der organischen Werkstoffe. R. 109, 49

Boersch, H., Nebenbilder in elektronenmikroskopischen Abbildungen. R. 109, 157

Borger, G., s. H. Bayerle

BraB, K., u. W. Schreier, Veränderungen der Baumwollzellulose durch Färbevorgänge. $0.108,155$

*) Ein Generalregister für die Bände 51-100 befindet sich in Vorbereitung. 